\title{
Special Issue for FAW 2014
}

\author{
Jianer Chen ${ }^{1}$ • John E. Hopcroft ${ }^{2}$
}

This Special Issue arises from the 8th International Frontiers of Algorithmics Workshop (FAW 2014), held in Zhang Jiajie, China, June 28-30, 2014. The International Frontiers of Algorithmics Workshop (FAW) provides a focused forum on current trends of research on algorithms, discrete structures, operation research, combinatorial optimization, and their applications. FAW brings together international experts at the research frontiers in these areas to exchange ideas and to present significant new results. The mission of FAW is to stimulate the various fields for which algorithmics can become a crucial enabler, and to strengthen the ties between the Eastern and Western algorithmics research communities as well as theory and practice of algorithmics. Submissions to FAW come from all countries over the world, with a significant portion from Europe and North America. Program committees of FAW consist of algorithmic researchers with international reputation, and keynote speeches at FAW are given by distinguished researchers, including a number of Turing Award Laureates.

FAW has run nine consecutive times since 2007. The years leading up to FAW 2014 had seen the conference series establish itself as a key contributor on the international scene, attracting the high quality of submission, playing an important reginal and international role, and promising to become a focused forum on current research trends on algorithms.

Submission invitations were sent to the best papers presented at FAW 2014 for inclusion in this Special Issue. After a thorough and rigorous review process, to the

\footnotetext{
$\bowtie \quad$ Jianer Chen

chen@cse.tamu.edu

John E. Hopcroft

jeh@cs.cornell.edu

1 Texas A\&M University, College Station, TX, USA

2 Cornell University, Ithaca, NY, USA
} 
usual high standards of JOCO, eight submissions were selected for inclusion in this Special Issue.

We would like to thank all authors for submitting their work to FAW 2014 and to this Special Issue. We thank our fellow Programme Committee members of FAW2014, and the many outside referees they called on, for the hard work and expertise which they have brought to the difficult selection process consequent on the high quality of submissions to the conference and to the Special Issue. We express our appreciation to the JOCO EIC, Professor Ding-Zhu Du, and the members of the Editorial Board of JOCO for their encouragement and advice throughout the preparation of this Special Issue. Of course FAW 2014 would not have been possible without the support of our sponsors: Central South University, China, and the National Natural Science Foundation of China (NSFC). We therefore gratefully acknowledge their help in the realization of FAW 2014 from which this Special Issue has arisen. 